\title{
Postpartum Endometritis
}

National Cancer Institute

\section{Source}

National Cancer Institute. Postpartum Endometritis. NCI Thesaurus. Code C111914.

Infection of the endometrium, decidua and/or myometrium occurring at any time

between birth and 42 days postpartum. 